\title{
Reversible Cerebral Vasoconstriction Syndrome with Intracranial Hypertension: Should Decompressive Craniectomy Be Considered?
}

\author{
Ségolène Mrozek ${ }^{a} \quad$ Laurent Lonjaret $^{a} \quad$ Aude Jaffre $^{b}$ \\ Anne-Christine Januel ${ }^{c}$ Nicolas Raposo ${ }^{b}$ Sergio Boetto ${ }^{d}$ \\ Jean-François Albucher ${ }^{b}$ Olivier Fourcade ${ }^{a} \quad$ Thomas Geeraerts $^{a}$ \\ ${ }^{a}$ Department of Anesthesiology and Intensive Care, University Hospital of Toulouse, \\ University Paul Sabatier-Toulouse 3, Toulouse, France; ${ }^{b}$ Department of Vascular \\ Neurology, University Hospital of Toulouse, University Paul Sabatier-Toulouse 3, \\ Toulouse, France; ' Department of Neuroradiology, University Hospital of Toulouse, \\ University Paul Sabatier-Toulouse 3, Toulouse, France; ${ }^{d}$ Department of Neurosurgery, \\ University Hospital of Toulouse, University Paul Sabatier-Toulouse 3, Toulouse, France
}

\section{Keywords}

Reversible cerebral vasoconstriction syndrome - Decompressive craniectomy · Intracerebral hemorrhage $\cdot$ Intracranial hypertension

\begin{abstract}
Background: Reversible cerebral vasoconstriction syndrome (RCVS) is a rare cause of intracerebral hemorrhage (ICH) causing intracranial hypertension. Methods: Case report. Results: We report a case of RCVS-related ICH leading to refractory intracranial hypertension. A decompressive craniectomy was performed to control intracranial pressure. We discuss here the management of RCVS with intracranial hypertension. Decompressive craniectomy was preformed to avoid the risky option of high cerebral perfusion pressure management with the risk of bleeding, hemorrhagic complications, and high doses of norepinephrine. Neurological outcome was good. Conclusion: RCVS has a complex pathophysiology and can be very difficult to manage in cases of intracranial hypertension. Decompressive craniectomy should probably be considered.




\section{Case Reports in Neurology}

\section{Introduction}

Reversible cerebral vasoconstriction syndrome (RCVS) has been only recently described as a single nosological entity [1]. It is defined as sudden thunderclap headache with reversible multifocal narrowing of cerebral arteries lasting 1-3 months with or without focal neurological symptoms $[1,2]$. The reversibility can only be confirmed with the evolution, and at presentation, the diagnosis is often presumed [2]. RCVS may be associated with major complications such as subarachnoid hemorrhage (SAH) (20-25\%) and ischemic or hemorrhagic stroke (30\%) [3]. Intracerebral hemorrhage (ICH) is reported in the literature in the context of RCVS, but the pathophysiology remains unclear [4]. Diagnosis and management of RCVS may be challenging. We report here a case of RCVS-related ICH leading to refractory intracranial hypertension. A decompressive craniectomy (DC) was performed to control intracranial pressure (ICP) with good outcome. This case scenario aims to describe the possible therapeutic options that have been discussed to control ICP in the context of RCVS.

\section{Case Report}

A 42-year-old woman with a history of chronic migraine presented to the emergency department for a sudden thunderclap headache after swimming. She regularly used nonsteroidal anti-inflammatory medication and ergotamine associated with caffeine for chronic headache. At admission she described diffuse, explosive, and unusual headache with spontaneous resolution in $8 \mathrm{~h}$. On initial examination, general and neurological clinical examination was normal. Arterial blood pressure was normal. The initial cerebral computed tomography (CT) scan without contrast was normal. The cerebrospinal fluid sampling obtained by lumbar puncture revealed presence of oxyhemoglobin with $290 \mathrm{red}$ blood cells $/ \mathrm{mm}^{3}$. The diagnosis of SAH was retained and a cerebral angiography scheduled for the next day. On day 1 , she presented severe unusual headache with arterial hypertension followed by aphasia and a right hemiplegia. Shortly afterward, her consciousness worsened requiring intubation and intravenous sedation. A cerebral CT scan was immediately performed and revealed a left rolandic ICH (Fig. 1). Cerebral angiography was performed showing a diffuse segmental narrowing of cerebral arteries without aneurism or arteriovenous malformation (Fig. 2). The diagnosis of RCVS with hemorrhagic complication was retained and intravenous nimodipine was introduced. The ICH was removed by a neurosurgeon and an ICP monitoring probe was inserted in the left frontal lobe. Initial ICP reading was $15 \mathrm{~mm} \mathrm{Hg}$. Intravenous sedation was continued in the intensive care unit. However, norepinephrine was required to keep cerebral perfusion pressure (CPP) over $60 \mathrm{~mm} \mathrm{Hg}$. Immediate postoperative transcranial Doppler (TCD) was normal. A control cerebral CT scan was performed the next day showing a residual intracranial hematoma and perilesional edema. Forty-eight hours later, ICP increased to above $30 \mathrm{~mm} \mathrm{Hg}$ despite optimization of medical treatment (head elevation, increased sedation, and osmotherapy). TCD readings for the right middle cerebral artery (MCA) were: pulsatility index at 1.6 and diastolic velocity at $23 \mathrm{~cm} / \mathrm{s}$, and for the left MCA: pulsatility index at 1.6 and diastolic velocity at $21 \mathrm{~cm} / \mathrm{s}$, probably corresponding to raised ICP. A new cerebral CT scan revealed similar findings. A left DC was performed facing refractory intracranial hypertension. Thereafter, ICP and TCD normalized and sedation was stopped in the following days. Mechanical ventilation was successfully weaned on day 7. The patient was discharged from the intensive care unit at day 14 with aphasia and right hemiplegia with progressive improvement from 3 months. She was independent at 6 months with a modified 
Rankin scale score of 2. Magnetic resonance imaging found no residual signs of RCVS at 3 months.

\section{Discussion}

This case report aims to focus on the specific management of intracranial hypertension in the context of RCVS. Specific diagnostic criteria for RCVS have been proposed [1, 5]. Calabrese et al. [1] and Ducros [5] have suggested the following criteria: acute and severe headache (thunderclap headache), with or without neurological deficit; exclusion of aneurismal $\mathrm{SAH}$; segmental and multifocal vasoconstriction on angiography; normal or subnormal cerebrospinal fluid (CSF), and resolution of angiographic abnormalities within 12 weeks. The diagnosis can only be confirmed a posteriori, when angiographic abnormalities have disappeared.

In a prospective study, Ducros et al. [3] reported only $12 \%$ of abnormalities on initial CT scan performed at a mean of $4.1(0-20)$ days after headache onset. Focal and cortical SAH has been described early in the course of RCVS in $20-34 \%$ of cases [6]. If CT scan is normal, lumbar puncture has to be performed for CSF examination. Most of the time in RCVS, CSF samplings are normal or with minor abnormalities such as pleocytosis of up to 15 white blood cells, small numbers of erythrocytes, and/or minimally elevated protein (less than $1 \mathrm{~g} / \mathrm{dL})[1,3]$. Conventional cerebral angiography is the gold standard to bring out the diffuse alternating pattern of vasodilatation and vasoconstriction or "string of beads" [1]. Magnetic resonance angiography or computed tomography angiography are recommended to diagnose RCVS and rule out aneurismal SAH and other differential diagnosis such as pituitary apoplexy, arterial dissection, or venous sinus thrombosis.

Intracranial hemorrhages in RCVS have been reported in cases or series [3, 4]. These complications occur mainly during the first week, whereas ischemic events occur later, generally during the second week [2, 7]. Intracranial hemorrhages include cortical SAH, ICH, and subdural hemorrhages. Ducros et al. [2] described in a large prospective cohort of 89 patients with RCVS that 30 patients (34\%) presented with intracranial hemorrhages and 5 (6\%) with cerebral infarction. The three types of hemorrhages were described, sometimes 2 or 3 for the same patients: 27 patients presented with cortical SAH (30\%), 11 with ICH $(12 \%)$, and 2 with a subdural hemorrhage (2\%). ICH could be located either in the lobar or in the deep regions or was sometimes multifocal [2]. Similar findings were observed by Singhal et al. [8] in a multicenter study of 139 patients with RCVS, with $34 \%$ of patients with SAH and $20 \%$ with lobar hemorrhages. Patients with RCVS and hemorrhagic manifestations have a significantly higher rate of persistent focal deficit (30 vs. $2 \%$ ) and cerebral infarction (13 vs. $2 \%$ ) than patients without hemorrhagic complications [2]. Two independent factors for bleeding in RCVS have been identified: female gender and history of migraine [2].

The pathophysiology of hemorrhagic complications in RCVS remains uncertain. Ducros et al. [2] reported that up to $17 \%$ of patients with hemorrhagic RCVS initially presented isolated headaches and no abnormality on cerebral CT scan, and only after cortical SAH, ICH and/or subdural hemorrhage were observed, suggesting that abnormal vasoconstriction precluded the hemorrhage phenomena. Moreover, early vasogenic cerebral edema due to small vessels dysfunction with acute disruption of the blood-brain barrier has also been observed, suggesting that small distal arteries injury occurs early followed by medium and large arteries injury [2]. Chen et al. [9] reported that maximal vasoconstriction in large arteries on magnetic resonance angiography and TCD occurred in the late phase of RCVS, only 
18-22 days after headache onset. Intracranial hemorrhage seems therefore to be the result of the vasospasm and not the trigger, as in aneurysmal SAH. Small vessel rupture or reperfusion injuries due to RCVS can produce cortical SAH or ICH [2]. Thereafter, severe vasoconstriction of larger vessels may lead to cerebral infarction [1]. High blood pressure and cerebrovascular autoregulatory mechanism disorders in narrowed vessels may also be involved in hemorrhagic complications [10]. In the present case, the use of norepinephrine could be discussed. Norepinephrine was infused to keep the CPP over $60 \mathrm{~mm} \mathrm{Hg}$ during sedation and mechanical ventilation after evacuation of ICH. In case of preserved cerebral autoregulation, cerebral blood flow (CBF) remains stable during norepinephrine infusion. In case of impaired autoregulation, CBF increases with blood pressure with a risk of hyperemic state [11]. It is unknown if norepinephrine may exacerbate cerebral vasoconstriction in RCVS and if it could be deleterious. Recently, a case of RCVS following administration of epinephrine has been reported in the setting of an anaphylactic reaction during antibiotic allergy testing [12]. Vasopressor-induced hypertension is recommended in the treatment of cerebral vasospasm after aneurismal SAH. However, the optimal CPP in case of RCVS is not known and the balance between low CPP with ischemic risk and high CPP with bleeding risk may be difficult to appreciate. In the present case, CPP was maintained between 60 and $70 \mathrm{~mm} \mathrm{Hg}$, corresponding to a range of pressure where cerebral autoregulation was present.

Calcium channel blockers (orally or intravenously), such as nimodipine, nifedipine, or verapamil, are used as first-line therapy in some centers in patients with RCVS because of the similarities with aneurismal SAH [13]. The risk for arterial hypotension should be considered, with decrease in CBF in regions with severe constricted cerebral arteries. Endovascular therapies with intra-arterial vasodilators and balloon angioplasty have also been used for severe segmental vasoconstriction in patients with neurological deficit [14]. Recently, intra-arterial nimodipine, verapamil, and milrinone have been shown to be safe and effective in RCVS, sometimes requiring repetitive treatments [15]. In the present case, nimodipine has been used after cerebral angiography and diagnosis.

As ICH was associated with a decrease in consciousness level, raised ICP was suspected. Hematoma removal was therefore performed to control ICP but failed to maintain ICP below $20 \mathrm{~mm} \mathrm{Hg}$ due to brain edema. DC has then been performed with success. ICP was thereafter below $20 \mathrm{~mm} \mathrm{Hg}$, allowing intravenous sedation withdrawal with good outcome. DC has been shown to be effective to control ICP in case of spontaneous ICH [16] but has not been described in the context of RCVS. In the present case, DC had the major advantage of allowing ICP decrease and avoiding the risky option of high CPP with the risk of bleeding and hemorrhagic complications.

\section{Conclusion}

Raised ICP can occur after RCVS. DC could be of interest in RCVS with intracranial hypertension to avoid the risky option of high CPP management with the risk of bleeding and high doses of norepinephrine.

\section{Statement of Ethics}

Informed consent was obtained from the patient. 


\section{Case Reports in Neurology}

C 2017 The Author(s). Published by S. Karger AG, Basel www.karger.com/crn

Mrozek et al: Reversible Cerebral Vasoconstriction Syndrome with Intracranial Hypertension: Should Decompressive Craniectomy Be Considered?

\section{Disclosure Statement}

The authors declare that they have no conflict of interest.

\section{References}

1 Calabrese LH, Dodick DW, Schwedt TJ, Singhal AB: Narrative review: reversible cerebral vasoconstriction syndromes. Ann Intern Med 2007;146:34-44.

2 Ducros A, Fiedler U, Porcher R, Boukobza M, Stapf C, Bousser MG: Hemorrhagic manifestations of reversible cerebral vasoconstriction syndrome: frequency, features, and risk factors. Stroke 2010;41:2505-2511.

-3 Ducros A, Boukobza M, Porcher R, Sarov M, Valade D, Bousser MG: The clinical and radiological spectrum of reversible cerebral vasoconstriction syndrome. A prospective series of 67 patients. Brain 2007;130:3091-3101.

4 Werring DJ: Reversible cerebral vasoconstriction syndrome and intracranial hemorrhage: some answers, many questions. Stroke 2010;41:2455-2456.

-5 Ducros A: Reversible cerebral vasoconstriction syndrome (in French). Presse Med 2010;39:312-322.

-6 Chen SP, Fuh JL, Wang SJ: Reversible cerebral vasoconstriction syndrome: current and future perspectives. Expert Rev Neurother 2011;11:1265-1276.

-7 Neel A, Guillon B, Auffray-Calvier E, Hello M, Hamidou M: Reversible cerebral vasoconstriction syndrome (in French). Rev Med Interne 2012;33:586-592.

-8 Singhal AB, Haji-Ali RA, Topcuoglu MA, Fok J, Bena J, Yang D, Calabrese LH: Reversible cerebral vasoconstriction syndromes: analysis of 139 cases. Arch Neurol 2011;68:1005-1012.

9 Chen SP, Fuh JL, Wang SJ, Chang FC, Lirng JF, Fang YC, et al: Magnetic resonance angiography in reversible cerebral vasoconstriction syndromes. Ann Neurol 2010;67:648-656.

10 Shah AK: Non-aneurysmal primary subarachnoid hemorrhage in pregnancy-induced hypertension and eclampsia. Neurology 2003;61:117-120.

11 Mascia L, Andrews PJ, McKeating EG, Souter MJ, Merrick MV, Piper IR: Cerebral blood flow and metabolism in severe brain injury: the role of pressure autoregulation during cerebral perfusion pressure management. Intensive Care Med 2000;26:202-205.

-12 Palma JA, Fontes-Villalba A, Irimia P, Garcia-Eulate R, Martinez-Vila E: Reversible cerebral vasoconstriction syndrome induced by adrenaline. Cephalalgia 2012;32:500-504.

13 Dodick DW: Reversible segmental cerebral vasoconstriction (Call-Fleming syndrome): the role of calcium antagonists. Cephalalgia 2003;23:163-165.

-14 Ioannidis I, Nasis N, Agianniotaki A, Katsouda E, Andreou A: Reversible cerebral vasoconstriction syndrome: treatment with multiple sessions of intra-arterial nimodipine and angioplasty. Interv Neuroradiol 2012;18:297-302.

15 Bouchard M, Verreault S, Gariepy JL, Dupre N: Intra-arterial milrinone for reversible cerebral vasoconstriction syndrome. Headache 2009;49:142-145.

16 Murthy JM, Chowdary GV, Murthy TV, Bhasha PS, Naryanan TJ: Decompressive craniectomy with clot evacuation in large hemispheric hypertensive intracerebral hemorrhage. Neurocrit Care 2005;2:258262. 


\section{Case Reports in Neurology}

www.karger.com/crn

Mrozek et al.: Reversible Cerebral Vasoconstriction Syndrome with Intracranial Hypertension: Should Decompressive Craniectomy Be Considered?

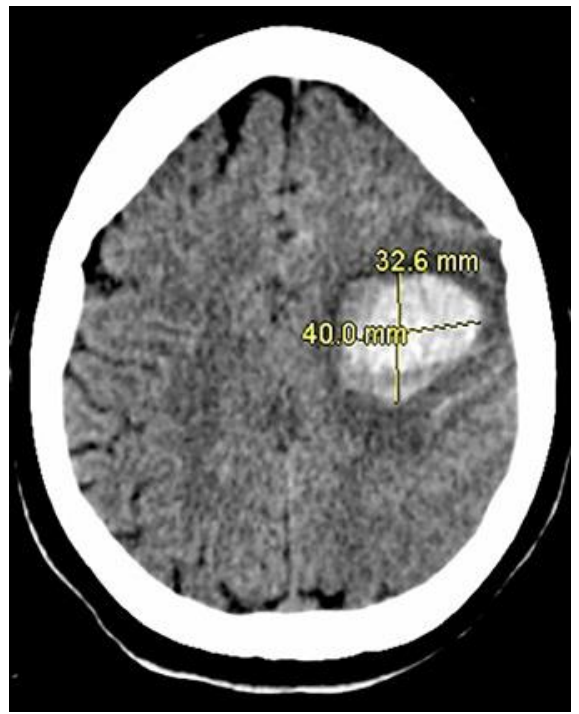

Fig. 1. Cerebral computed tomography scan on day 1 after occurrence of aphasia and right hemiplegia revealing a left rolandic intraparenchymal hematoma.

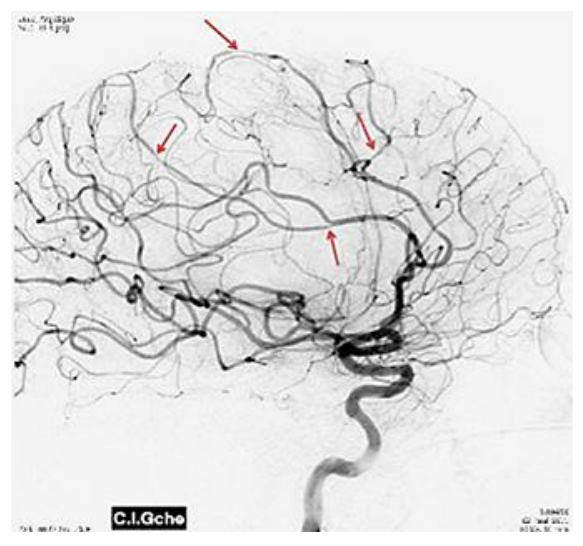

Fig. 2. Cerebral angiography on day 1. Issue by left arterial carotid showing segmental narrowing and dilatation (string of beads) in all vascular territories without aneurysm (red arrows). 